\section{(2) OPEN ACCESS}

\title{
Case-finding and genetic testing for familial hypercholesterolaemia in primary care
}

\author{
Nadeem Qureshi (D) , ${ }^{1}$ Ralph Kwame Akyea (D) ,' Brittany Dutton,' \\ Steve E Humphries, ${ }^{2}$ Hasidah Abdul Hamid, ${ }^{1,3}$ Laura Condon, ${ }^{1}$ Stephen F Weng, ${ }^{4}$ \\ Joe Kai, ${ }^{1}$ for the FAMCAT study
}

\begin{abstract}
- Additional supplemental material is published online only. To view, please visit the journal online (http://dx.doi. org/10.1136/heartjnl-2021319742)
\end{abstract}

${ }^{1}$ Centre for Academic Primary Care, School of Medicine, University of Nottingham, Nottingham, UK ${ }^{2}$ Centre for Cardiovascular Genetics, Institute of Cardiovascular Science, University College London, London, UK

${ }^{3}$ Department of Primary Care Medicine, Faculty of Medicine, Universiti Teknologi MARA, Sungai Buloh, Malaysia ${ }^{4}$ Associate Director Cardiovascular and Metabolism, Janssen Research \& Development, High Wycombe, UK

\section{Correspondence to} Professor Nadeem Qureshi, Centre for Academic Primary Care, School of Medicine, University of Nottingham, Nottingham NG7 2RD, UK; nadeem.qureshi@nottingham. ac.uk

NQ and RKA contributed equally.

SFW and JK contributed equally.

Received 21 May 2021 Accepted 25 August 2021 Published Online First 14 September 2021

\section{Linked}

- http://dx.doi.org/10.1136/ heartjnl-2021-320085

Check for updates

(c) Author(s) (or their employer(s)) 2021. Re-use permitted under CC BY. Published by BMJ.

To cite: Qureshi N, Akyea RK, Dutton $\mathrm{B}$, et al. Heart

2021:107:1956-1961.

\section{ABSTRACT \\ Objective Familial hypercholesterolaemia $(\mathrm{FH})$ is} a common inherited disorder that remains mostly undetected in the general population. Through FH casefinding and direct access to genetic testing in primary care, this intervention study described the genetic and lipid profile of patients found at increased risk of FH and the outcomes in those with positive genetic test results. Methods In 14 Central England general practices, a novel case-finding tool (Familial Hypercholetserolaemia Case Ascertainment Tool, FAMCAT1) was applied to the electronic health records of 86219 patients with cholesterol readings (44.5\% of total practices' population), identifying 3375 at increased risk of FH. Of these, a cohort of 336 consenting to completing Family History Questionnaire and detailed review of their clinical data, were offered $\mathrm{FH}$ genetic testing in primary care.

Results Genetic testing was completed by 283 patients, newly identifying 16 with genetically confirmed $\mathrm{FH}$ and 10 with variants of unknown significance. All 26 (9\%) were recommended for referral and 19 attended specialist assessment. In a further 153 (54\%) patients, the test suggested polygenic hypercholesterolaemia who were managed in primary care. Total cholesterol and low-density lipoprotein-cholesterol levels were higher in those patients with $\mathrm{FH}$-causing variants than those with other genetic test results ( $p=0.010$ and $p=0.002$ ).

Conclusion Electronic case-finding and genetic testing in primary care could improve identification of $\mathrm{FH}$; and the better targeting of patients for specialist assessment. A significant proportion of patients identified at risk of $\mathrm{FH}$ are likely to have polygenic hypercholesterolaemia. There needs to be a clearer management plan for these individuals in primary care.

Trial registration number NCT03934320.

\section{INTRODUCTION}

Familial hypercholesterolaemia $(\mathrm{FH})$ is one of the most common inherited disorders. The heterozygote form of $\mathrm{FH}$ is estimated to have a prevalence of 1 in $250 .^{1}$ Left untreated, these individuals are at increased risk of premature coronary heart disease (CHD)..$^{2-4}$ Treatment with lipid-lowering treatment, like statins, can dramatically reduce this risk. ${ }^{3}$ However, the majority of patients remain undiagnosed and untreated. ${ }^{267}$

International guidelines recommend the identification of $\mathrm{FH}$ using various case-finding tools. ${ }^{2} 89$ In the UK, the 2008 National Institute for Health and Care Excellence (NICE) FH guidelines recommended identifying possible $\mathrm{FH}$ cases in primary care using the Simon-Broome (S-B) criteria. $^{6}$ In addition to the established case-finding approaches, we have developed a novel case-finding tool (Familial Hypercholetserolaemia Case Ascertainment Tool, FAMCAT). ${ }^{10}$

Once an individual is identified at possible risk of $\mathrm{FH}$, guidelines recommend the individual should be genetic tested to confirm the diagnosis. ${ }^{9} 11$ Currently, this testing is not offered in primary care but in specialist care. ${ }^{12}$ The most recently developed genetic test for $\mathrm{FH}$ uses next-generation sequencing (NGS) diagnostic assay. In addition to identifying $\mathrm{FH}$-causing variants, this more comprehensive analysis identifies individuals with genetic variants of unknown significance (VUS) and those with minor variants that collectively can suggest the individual has a polygenic cause for hypercholesterolaemia. ${ }^{13}$

We offered direct access to $\mathrm{FH}$ genetic testing in primary care, as part of a study to optimise the FAMCAT case-finding tool. We sought to identify the genetic and lipid profile of patients found at increased risk of $\mathrm{FH}$, and the outcomes in those identified with $\mathrm{FH}$-causing genetic variants or VUS in primary care.

\section{METHODS}

\section{Study population and procedure}

In this multicentre, non-randomised, intervention study, 14 general practices, in the catchment areas of two UK lipid specialist services, expressed an interest to participate from May 2017 to November 2019, with a total practice population of 193589 . A range of urban, rural and suburban areas were invited to take part in the study based on their English index of multiple deprivation score, an official measure of material deprivation, to ensure a diverse practices were recruited. ${ }^{14}$

Participating general practices were given a 1 hour face-to-face introductory session on identifying $\mathrm{FH}$ based on NICE FH guideline, and demonstration of how to use the FAMCAT FH case-finding tool to perform electronic search of patient records and identify eligible patients for the study. The FH casefinding tool was then installed on all general practice computers. Fifty study participation packs, for patients, were despatched to each practice.

Nominated practice administrators first performed electronic health record search to identify patients, aged 18 years and above, with 
a cholesterol reading $(n=86219$ or $44.5 \%$ of total practices' population). The FAMCAT1 case-finding tool was applied to these patients' records by the practice administrator (based on the lower threshold of FAMCAT1 algorithm: probability of FH of 1 in 500). ${ }^{10}$ This identified 3375 at possible risk of $\mathrm{FH}$ of whom the first 336 consenting to completion of Family History Questionnaire (FHQ), researcher access to their clinical records and genetic testing in their practice, were recruited.

The research assistant notified the practice administrator which of the recruited patients to invite for genetic tests at phlebotomy clinics in the practice. In line with NICE FH guideline, a repeat cholesterol test was performed at the same time. As part of the invitation, participants were given an information leaflet describing the nature of the test. The genetic test was conducted by the Bristol NHS FH Genetic Laboratory (CPA Ref: 2907) using a NGS diagnostic assay. A simplified summary of the genetic test results was sent to both the participant and their nominated general practitioner (GP).

Participants were eligible if they were registered with a participating general practice, able to give written informed consent, aged 18 years or over, had a serum cholesterol recorded in their electronic health records and without a previous diagnosis of $\mathrm{FH}$.

\section{Clinical data}

Clinical data on the recruited participants were collected at baseline through automated electronic healthcare record extraction, manual review of electronic health records by a research assistant and self-reported FHQ of CHD. ${ }^{15}$

\section{Outcomes}

\section{Genetic outcome measures}

Genetically confirmed $F H$ was defined as carrying a definitive pathogenic variant in LDLR (18 exons), APOB (28 exons), PCSK9 (12 exons) and homozygosity in LDLRAP1 (9 exons) as reported on the laboratory test report.

VUS are variants that cannot be definitively classified as pathogenic or benign. All identified variants were independently assessed by three senior experts in $\mathrm{FH}$ genetic testing (MWclinician scientist, SEH-cardiovascular geneticist, AW-lipidologist) using internationally agreed criteria published by the American College of Medical Genetics guidelines. ${ }^{16}$ These assessors determined if variant classifications are 'definitely not' and 'likely not pathogenic' (class 1 and 2), 'VUS' (class 3) and 'likely' and 'definitely pathogenic' (class 4 and 5). The assessors had not been involved in the design of the project or the analysis of these data.

Polygenic hypercholesterolaemia risk score (PHR score) is based on the combined weighted effect of 12 common lowdensity lipoprotein (LDL)-cholesterol raising single nucleotide polymorphisms (SNPs) in 11 genes. ${ }^{13}$ Full details of the SNPs are provided in online supplemental table 1 . Using a weighted score, each individual was assigned to the appropriate decile. ${ }^{13}$ With a PHR score in decile 1-3 designated as a low likelihood of polygenic hypercholesterolaemia, a 6th-10th decile score designated as a high likelihood of polygenic hypercholesterolaemia and 4th and 5 th decile score as intermediate likelihood. ${ }^{17}$

Those patients with FH confirmed on genetic test or with unclear results (VUS) were recommended for specialist referral after further discussion with their GP. If the test did not identify FH-causing variant but only indicated a high polygenic hypercholesterolaemia score, GP was informed of patient's predisposition to raised cholesterol and recommended that they are recalled for cardiovascular risk assessment and patient advised that the practice will be in touch to arrange a cardiovascular health check. Finally, remaining patients were informed of the negative genetic test results and sent a healthy lifestyle leaflet.

\section{Biochemical outcome measures}

All available information on lipid profiles of participants (total cholesterol, LDL-cholesterol, HDL-cholesterol and triglycerides) were extracted from patients' records. The latter included lipoprotein(a) (Lp(a)) levels when available in the local laboratory.

\section{Process measures}

This included the participants' recruitment rate as defined by participant completing FHQ. Following genetic testing, we also calculated the referral rate and noted participants attending specialist referral. Also, from reviewing hospital medical records of patients seen in specialist care, we identified outcome of clinical assessment and those patients offered cascade testing to relatives.

\section{Patient and public involvement}

Our patient and public co-applicant was involved in the design and conduct of this study. The patient representatives participated in interpreting the study results and assisted with the plain English summary.

\section{Statistical analysis}

Primary outcome and process measures were presented descriptively. Continuous normally distributed variables were described by the mean and SD while continuous non-normally distributed variables were described by the median and IQR. Categorical variables presented as count and percentage. Wilcoxon rank-sum test for continuous data and Fisher's exact test for categorical data were used to compare cholesterol profiles and statin prescribing between groups.

In order to complete 300 genetic tests, we planned to recruit 345 patients, assuming 13\% of patient will not complete genetic testing.

\section{RESULTS}

The 14 recruited general practices' index of deprivation ranged from 1 (most deprived) to 10 (least deprived) with the median deprivation score of 7 (IQR 6.25) and 5 general practices with scores below 5. Further details on the recruited practices are presented in online supplemental table 2.

As indicated in figures 1 and 2, of 336 consenting patients at risk of $\mathrm{FH}, 283(84.2 \%)$ attended for genetic testing in primary care and $26(9 \%)$ of the 283 participants tested were recommended referral for specialist assessment (16 with a confirmed FH-causing variant and 10 with VUS). During the study period, one of these patients, with genetically confirmed $\mathrm{FH}$, died from cardiovascular disease. Full details of the variants that were identified are listed in online supplemental tables 3 and 4 . All identified variants were reviewed by three experts. They agreed that all of the FH-causing variants were correct, and that none of VUS variants could be reclassified as pathogenic.

Of the 26 advised referral for FH-causing variant or with VUS, by completion of the study, 19 were seen by specialists (figure 2 ) . Of the 13 with $\mathrm{FH}$-causing variants (genetically confirmed $\mathrm{FH}$ ) seen by specialist, cascade screening to relatives was commenced in 8 families. The other six patients, with VUS results were managed based on their clinical phenotype and two of these were offered cascade screening. Additional details on process 


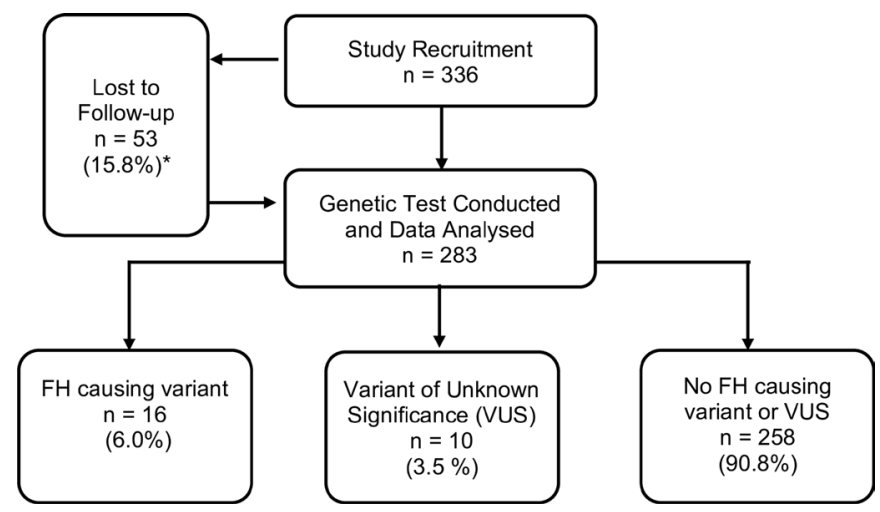

Figure 1 Study flow diagram. FH, familial hypercholesterolaemia. * Lost to follow up includes: 42 participants did not respond to genetic test invite and 11 left the practice before genetic test invite was sent.

outcomes are given in online supplemental table 5. A further 153 participants (54.1\% of those tested) had a high polygenic hypercholesterolaemia score (deciles 6-10). The decile distribution of the whole sample is presented in online supplemental table 6 and online supplemental figure 1.

Electronic health records were available for 260 of the 283 participants who had had genetic testing. These 260 participants had a similar age-sex profile to the 76 that were lost to follow-up (online supplemental table 7). The lipid profile demonstrated raised cholesterol levels with 175 (67\%) patients fulfilling S-B cholesterol criteria (table 1). Further in 93 of the participants, $\mathrm{Lp}(\mathrm{a})$ levels were available with a median of 156.8 (IQR 85-492) and 28 (10.8\%) with Lp(a) above reference range $(>300 \mathrm{mmol} / \mathrm{L})$. Although no arcus cornealis was identified in general practice records, 3 of the 19 patients, seen in hospital, were found by the specialist to have arcus cornealis. No patient was found to have tendon xanthoma.

Table 2 compares lipid profiles and statin prescribing by the nature of genetic test result where electronic health records were available to review $(n=260)$. Prior to the genetic diagnosis, all subgroups had raised median total and LDL-cholesterol
Table 1 Profile of study participants where manual electronic health records available $(n=260)$

\begin{tabular}{ll}
\hline Variable & $\begin{array}{l}\text { Participants with no } \\
\text { previous FH diagnosis } \\
(\mathbf{n}=260)\end{array}$ \\
\hline Age in years, mean (SD) & $56.3(11.4)$ \\
\hline $\begin{array}{l}\text { Females, } \mathrm{n}(\%) \\
\text { Highest total cholesterol level, median (IQR) }\end{array}$ & $7.6(6.7-8.2)$ \\
\hline Highest LDL-cholesterol level, median (IQR) & $5.0(4.2-5.6)$ \\
$\begin{array}{l}\text { On statin at the time of highest total cholesterol } \\
\text { measurement, } \mathrm{n}(\%)\end{array}$ & $66(25.4)$ \\
\hline $\begin{array}{l}\text { On high-potency statin at the time of highest total } \\
\text { cholesterol measurement, } \mathrm{n}(\%)\end{array}$ & $17(6.5)$ \\
\hline Ever total cholesterol $>7.5$ or $\mathrm{LDL}>4.9, \mathrm{n}(\%)$ & $175(67.3)$ \\
\hline Number with Lp(a) record (\%) & $93(35.8)$ \\
\hline $\mathrm{L}(\mathrm{a})$ level, median (IQR) & $156.8(85-492)$ \\
\hline Examined for arcus cornealis, $\mathrm{n}(\%)$ & 0 \\
\hline Examined for tendon xanthoma, $\mathrm{n}(\%)$ & 0 \\
\hline
\end{tabular}

$\mathrm{FH}$, familial hypercholesterolaemia; LDL, low-density lipoprotein; Lp(a), lipoprotein(a).

concentrations, with those genetically confirmed to have $\mathrm{FH}$ having higher median values than those with other test results. The median cholesterol values in those with a VUS was similar to those with no $\mathrm{FH}$-causing genetic variant and those with a high PHR score (in the 6th-10th decile). Also, a higher proportion of the genetically confirmed FH group were prescribed a statin compared with other groups. Two participants were on high-potency stains at the time of the highest ever cholesterol, both noted before the start of the study. Further comparison of absolute differences in cholesterol concentrations and statin prescribing is presented in online supplemental table 8 .

\section{DISCUSSION} Key findings

In patients with no previous diagnosis of $\mathrm{FH}$, from a general primary care population, 26 of 283 completing genetic testing

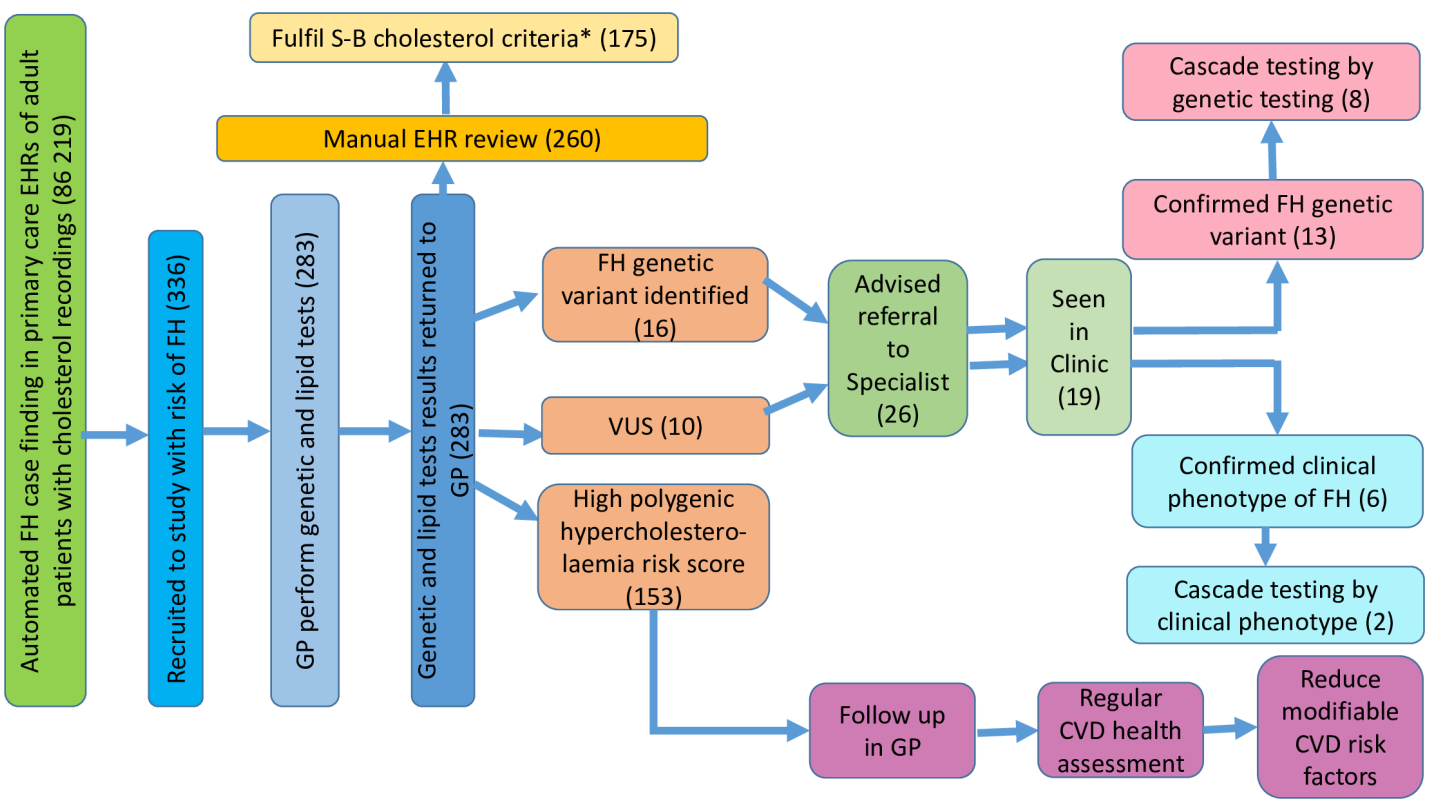

Figure 2 Graphical display of key findings. *Ever had total cholesterol $>7.5$ or LDL-C $>4.9 \mathrm{mmol} / \mathrm{L}$. CVD, cardiovascular disease; EHR, electronic health record; FH, familial hypercholesterolaemia; GP, general practitioner; S-B, Simon-Broome; VUS, variants of unknown significance. 
Table 2 Current cholesterol profile and statin prescribing by reported genetic test results in 260 participants with electronic health records available

\begin{tabular}{|c|c|c|c|c|}
\hline & $\begin{array}{l}\text { Genetically confirmed } \\
\text { FH } \\
(n=16)\end{array}$ & $\begin{array}{l}\text { VUS } \\
(n=9)\end{array}$ & $\begin{array}{l}\text { High PHR score } \\
(n=139 *)\end{array}$ & $\begin{array}{l}\text { No genetic mutation } \\
(n=98)\end{array}$ \\
\hline Age in years, mean (SD) & $55.8(13.7)$ & $60.2(13.9)$ & $56.6(11.7)$ & $55.9(10.5)$ \\
\hline Females, n (\%) & $10(62.5)$ & $9(100)$ & $38(27.3)$ & $62(63.3)$ \\
\hline Highest ever total cholesterol, median mmol/L (IQR) & $8.6(6.9-11.5)$ & $7.6(6.1-8.0)$ & $7.7(6.8-8.3)$ & $7.5(6.8-7.9)$ \\
\hline Highest ever LDL-cholesterol, median mmol/L (IQR) & $6.4(4.4-8.5)$ & $4.7(3.6-5.6)$ & $5.1(4.4-5.7)$ & $4.8(4.0-5.3)$ \\
\hline On statin at time of highest cholesterol record, $\mathrm{n}(\%)$ & $11(68.8)$ & $2(22.2)$ & $34(24.5)$ & 19 (19.4) \\
\hline On high-potency statin at time of highest cholesterol record, $\mathrm{n}(\%)$ & $2(12.5)$ & $1(11.1)$ & $9(6.5)$ & $5(5.1)$ \\
\hline On statin at time of highest cholesterol record after study start, $\mathrm{n}(\%)$ & $6(37.5)$ & $2(22.2)$ & 19 (13.7) & $10(10.2)$ \\
\hline On high-potency statin at time of highest cholesterol record after study start, $\mathrm{n}(\%)$ & 0 & $1(11.1)$ & $6(4.3)$ & $3(3.1)$ \\
\hline Ever total cholesterol $>7.5$ or $\mathrm{LDL}>4.9, \mathrm{n}(\%)$ & $12(75.0)$ & $6(66.7)$ & $101(72.7)$ & $58(59.2)$ \\
\hline Number with $L p(a)$ record $(\%)$ & $6(37.5)$ & 3 & 50 & 35 \\
\hline Lp(a) level, median (IQR) & $157.6(142-177)$ & $83(83-100)$ & $165(89-253.5)$ & $150(85-791)$ \\
\hline Examined for arcus cornealis, $\mathrm{n}(\%)$ & 0 & 0 & 0 & 0 \\
\hline Examined for tendon xanthoma, $\mathrm{n}(\%)$ & 0 & 0 & 0 & 0 \\
\hline
\end{tabular}

${ }^{*}$ This includes two patients with VUS who had high polygenic risk scores.

FH, familial hypercholesterolaemia; LDL, low-density lipoprotein; Lp(a), lipoprotein(a); PHR, polygenic hypercholesterolaemia risk; VUS, variants of unknown significance.

required specialist referral. Two-thirds (16) of these individuals had genetically confirmed $\mathrm{FH}$, equating to 1 in 18 of those tested. A further $53 \%$ of the 283 participants tested were identified with a polygenic inherited predisposition to hypercholesterolaemia (PHR score in the 6th-10th decile).

Total cholesterol and LDL levels, as well as statin prescribing, were higher in those patients identified with newly diagnosed genetically confirmed $\mathrm{FH}$. As study participants were identified using a FH case-finding tool, it is not surprising their cholesterol levels were higher than the general population. In some genetically confirmed patients, the clinical sequelae of $\mathrm{FH}$, specifically arcus cornealis, was not documented in primary care records but noted on specialist clinical assessment.

\section{Relationship to other literature}

This is the first study to introduce genetic testing in primary care to examine and confirm the genetic profile of people identified at risk of having $\mathrm{FH}$ from the general population, as part of routine clinical care. Previous studies on FH assessment in primary care have included intervention to identify patients at risk of FH using clinical diagnostic criteria and referring them to specialist lipid services for confirmatory diagnosis with genetic testing. ${ }^{18-21}$

Our previous study of cardiovascular genetic testing using postal DNA saliva collection ${ }^{22}$ was acceptable to patients. ${ }^{23}$ The current study collecting genetic blood samples in primary care appears to be similarly acceptable. As well as identifying individuals with $\mathrm{FH}$-causing genetic variants, this study identified those with polygenic hypercholesterolaemia. There is increasing interest in polygenic causes of common chronic diseases, including both hypercholesterolaemia and CHD. ${ }^{17}{ }^{24}$ However, it remains unclear how this information can be used in healthcare either as a standalone risk assessment or as part of a multifactorial risk score. ${ }^{25}$

In this study, we newly identified 16 patients with genetically confirmed FH from 14 general practices, based on genetic testing 283 patients from an automated primary care record search of 86219 individuals with cholesterol readings already recorded in their medical records. This compares favourably with $\mathrm{FH}$ screening in children, with 20 identified with genetically confirmed FH from 92 general practices based on cholesterol testing 10118 children at immunisation and genetic testing 92 children, over cholesterol threshold. ${ }^{26}$ Currently, case-finding in adults fits more closely into the infrastructure of primary care and is more resource efficient. This does not negate the value of screening children for $\mathrm{FH}$, but identifying all cases of $\mathrm{FH}$ will require a multifaceted approach.

Following identification of index cases with genetically confirmed FH, the next step is to cascade FH screening to other relatives with around $50 \%$ of first-degree relatives affected. ${ }^{9} 11$ It is estimated that each index case identifies on average another 1.65 relatives with the condition. ${ }^{27}$ From these 14 general practices, by the end of the study period, cascade screening had already started in 8 families of genetically confirmed index cases.

\section{Strengths and weaknesses}

Routine cholesterol measurement, as recommended by national FH guidelines, is established in primary care. In this study, we were also able to integrate genetic testing within the infrastructure of the general practice phlebotomy services using blood bottles readily available in primary care (EDTA specimen bottles most commonly used for full blood count and glycosylated haemoglobin). Furthermore, this study actively recruited diverse general practices, including 36\% participating from socially deprived areas.

Given the pragmatic nature of the study and, for ethical reasons, the research team could not identify patients to recruit prior to written consent. As a consequence, practices invited some patients who did not fulfil the study eligibility criteria (FAMCAT probability threshold 1 in 500). In future studies and $\mathrm{FH}$ case-finding tool development, the selection of patients will be further automated to ensure only eligible patients are recruited.

\section{Clinical implications}

The starting point for identification of FH in primary care is the availability of serum cholesterol levels. In this study, nearly $45 \%$ of the adult patients registered with the practices had a result. Periodic health checks in primary care offer the opportunity to increase the proportion of the population tested. ${ }^{28}$ In England, this is offered around every 5 years for the national vascular 
check programme. ${ }^{29}$ The study also demonstrated genetic testing for inherited hypercholesterolaemia can be performed in primary care. This could lead to refinement of the FH referral pathway with only those with genetically confirmed $\mathrm{FH}$ and those with strong clinical phenotype of FH referred to specialists. This could reduce unnecessary referrals and workload. In addition to $\mathrm{FH}$, other inherited monogenic lipid disorders may be identified, for example, familial combined hyperlipidaemia. More comprehensive primary care pathways to identify several inherited lipid disorders may need to be considered.

One key challenge with current state-of-the-art genetic testing is the increased diagnostic uncertainty when analysing samples using NGS. In this study, $4 \%$ of those tested were identified with VUS. These participants also needed referral, but it could be challenging for GPs to explain the diagnostic uncertainty and need for specialist assessment to confirm FH or a 'benign' result. A significant proportion of these participants will then still remain classified as VUS and may return to their GPs for further clarification. Within this group, there will be patients who have the clinical features of (monogenic) FH but no definitive genetic diagnosis. In some cases, these patients will be diagnosed with polygenic hypercholesterolaemia. Specialists will still manage them aggressively with high potency statins.

As found in this study, NGS can also identify polygenic hypercholesterolaemia. Patients with these findings are unlikely to require specialist referral but this novel risk factor could contribute to multifactorial cardiovascular risk assessment, such as, Framingham or QRisk score. If this or other genetic tests are to be introduced into primary care, practitioners would need to be literate in understanding test results and explaining these findings to patients. This may require outreach specialist nurse support. A recent review recommended the need for multidisciplinary clinics involving both primary and specialist care. ${ }^{30}$

\section{Research implications}

This study indicates a randomised controlled study design, measuring clinically relevant outcome measures, such as, the proportion of individuals with genetically confirmed $\mathrm{FH}$ achieving optimal cholesterol level, is needed to provide robust

\section{Key messages}

What is already known on this subject?

- Familial hypercholesterolaemia (FH) is currently underdiagnosed and undertreated.

- Clinical criteria and electronic tools exist for FH case-finding in primary care.

- Genetic testing for FH is not currently offered in primary care.

\section{What might this study add?}

- This study demonstrates that undiagnosed FH cases can be identified from the general population in primary care, using electronic case finding and genetic testing.

- Less severe polygenic hypercholesterolaemia is also found in a large proportion of patients identified at risk of $\mathrm{FH}$.

\section{How might this impact on clinical practice?}

- The integration of genetic testing with $\mathrm{FH}$ case finding in primary care electronic health records could refine current referral pathways, leading to more appropriate use of specialist services for management of genetically confirmed $\mathrm{FH}$ and cascade genetic testing to relatives. evidence on whether to introduce a combined approach using an $\mathrm{FH}$ case-finding tool with genetic testing in general practice. This intervention would be compared with usual practice of case-finding without genetic testing. Prior to a full trial, a pilot randomised study will be needed to estimate appropriate sample size. The cardiovascular risk associated with polygenic hypercholesterolaemia in the general population needs further exploration. The utility of incorporating the polygenic hypercholesterolaemia score into cardiovascular risk assessment tools should also be considered.

\section{Conclusions}

Case finding for patients at increased risk of $\mathrm{FH}$, combined with genetic testing, can be performed in primary care. This could help enhance the referral pathway to specialist care. This identifies genetically confirmed FH and uncertain test results and polygenic hypercholesterolaemia. The complexity of genetic test results reported indicates a need for outreach specialist support to interpret the test findings and advise on care pathways. A significant proportion of those identified with possible $\mathrm{FH}$ on case finding will have polygenic hypercholesterolaemia. The cardiovascular risk and management associated with this still needs to be clarified.

Acknowledgements The authors would like to thank members of the FAMCAT external study steering committee (Paul Roderick, Dermot Neely, Andrew Neil, Simon Williams) and other study investigators (Matthew Jones, Kate Walters, Katherine Payne, Barbara Hanratty) for their guidance and patient representatives Phil Rowlands and Mark Fishers for their advice. We would like to thank the project administrators (Stephanie Gallimore \& Christina Sheehan) for their meticulous work to keep the project on track. We also thank the local lipidologists (Pankaj Gupta, Roger Stanworth) for their specialist input, Tony Wierzbicki and Maggie Williams for reviewing genetic test results. SEH acknowledges grants RG3008 and PG008/08 from the British Heart Foundation, and the support of the UCLH NIHR BRC. FAMCAT study team acknowledges the support of the National Institute for Health Research Clinical Research Network (NIHR CRN). Finally, we thank all the patients and primary care practices for their participation in this study.

Contributors NQ and JK secured funding. NQ, SW and JK conceptualised the study design and methods, and provided primary care interpretation of findings. NQ and BD managed the study. BD, RKA and SW conducted the analysis. NQ wrote the first draft. All study authors have contributed to interpretation, revising, writing and finalising the final submission version of the manuscript.

Funding This study/project is funded by the National Institute for Health Research (NIHR) School for Primary Care Research (project reference FR12-332).

Competing interests NQ has received honoraria and travel costs for lectures from AMGEN and grants from National Institute for Health Research Health Technology Assessment Programme during the conduct of the study, SW reports personal fees from AMGEN, personal fees from Quealth outside the submitted work and was a member of Clinical Practice Research Datalink Independent Scientific Advisory Committee (ISAC). SEH reports grants from British Heart Foundation during the conduct of the study.

\section{Patient consent for publication Not required.}

Ethics approval The study was sponsored by the University of Nottingham (sponsor reference: 16090), with approvals granted by the Health Research Authority (HRA, IRAS project ID: 213043) after ethics review by NRES Committee East Midlands (reference: 16/EM/0461).

Provenance and peer review Not commissioned; externally peer reviewed.

Data availability statement No data are available. We do not have consent from participants to share their data for the purposes of future research.

Supplemental material This content has been supplied by the author(s). It has not been vetted by BMJ Publishing Group Limited (BMJ) and may not have been peer-reviewed. Any opinions or recommendations discussed are solely those of the author(s) and are not endorsed by BMJ. BMJ disclaims all liability and responsibility arising from any reliance placed on the content. Where the content includes any translated material, BMJ does not warrant the accuracy and reliability of the translations (including but not limited to local 
regulations, clinical guidelines, terminology, drug names and drug dosages), and is not responsible for any error and/or omissions arising from translation and adaptation or otherwise.

Open access This is an open access article distributed in accordance with the Creative Commons Attribution 4.0 Unported (CC BY 4.0) license, which permits others to copy, redistribute, remix, transform and build upon this work for any purpose, provided the original work is properly cited, a link to the licence is given, and indication of whether changes were made. See: https://creativecommons.org/ licenses/by/4.0/.

ORCID iDs

Nadeem Qureshi http://orcid.org/0000-0003-4909-0644

Ralph Kwame Akyea http://orcid.org/0000-0003-4529-8237

\section{REFERENCES}

1 Akioyamen LE, Genest J, Shan SD, et al. Estimating the prevalence of heterozygous familial hypercholesterolaemia: a systematic review and meta-analysis. BMJ Open 2017;7:e016461.

2 Nordestgaard BG, Chapman MJ, Humphries SE, et al. Familial hypercholesterolaemia is underdiagnosed and undertreated in the general population: guidance for clinicians to prevent coronary heart disease: consensus statement of the European Atherosclerosis Society. Eur Heart J 2013;34:3478-90.

3 Humphries SE, Cooper JA, Seed M, et al. Coronary heart disease mortality in treated familial hypercholesterolaemia: update of the UK Simon Broome FH register. Atherosclerosis 2018:274:41-6.

4 lyen B, Qureshi N, Weng S, et al. Sex differences in cardiovascular morbidity associated with familial hypercholesterolaemia: a retrospective cohort study of the UK Simon Broome register linked to national Hospital records. Atherosclerosis 2020;315:131-7.

5 Besseling J, Hovingh GK, Huijgen R, et al. Statins in Familial Hypercholesterolemia: Consequences for Coronary Artery Disease and All-Cause Mortality. J Am Coll Cardiol 2016:68:252-60.

6 Qureshi N, Humphries SE, Seed M, et al. Identification and management of familial hypercholesterolaemia: what does it mean to primary care? Br J Gen Pract 2009;59:773-8

7 Abul-Husn NS, Manickam K, Jones LK, et al. Genetic identification of familial hypercholesterolemia within a single U.S. health care system. Science 2016:354:aaf7000.

8 Goldberg AC, Hopkins PN, Toth PP, et al. Familial hypercholesterolemia: screening, diagnosis and management of pediatric and adult patients: clinical guidance from the National Lipid Association Expert Panel on Familial Hypercholesterolemia. J Clin Lipidol 2011;5:S1-8.

9 National Institute for Health and Care Excellence. Familial hypercholesterolaemia: identification and management Clinical guideline [CG71]. Available: https:// wwwniceorguk/guidance/cg71 [Accessed 8 Mar 2021]

10 Weng S, Kai J, Akyea R, et al. Detection of familial hypercholesterolaemia: external validation of the FAMCAT clinical case-finding algorithm to identify patients in primary care. Lancet Public Health 2019;4:e256-64.

11 Watts GF, Sullivan DR, Hare DL, et al. Integrated guidance for enhancing the care of familial hypercholesterolaemia in Australia. Heart Lung Circ 2021;30:324-49.
12 National Health Service. National genomic test directory, 2018. Available: https:// wwwenglandnhsuk/publication/national-genomic-test-directories/ [Accessed $1 \mathrm{Apr}$ 2021].

13 Talmud PJ, Shah S, Whittall R, et al. Use of low-density lipoprotein cholesterol gene score to distinguish patients with polygenic and monogenic familial hypercholesterolaemia: a case-control study. Lancet 2013;381:1293-301.

14 Abel GA, Barclay ME, Payne RA. Adjusted indices of multiple deprivation to enable comparisons within and between constituent countries of the UK including an illustration using mortality rates. BMJ Open 2016;6:e012750.

15 Qureshi N, Bethea J, Modell B, et al. Collecting genetic information in primary care: evaluating a new family history tool. Fam Pract 2005;22:663-9.

16 Richards S, Aziz N, Bale S, et al. Standards and guidelines for the interpretation of sequence variants: a joint consensus recommendation of the American College of Medical Genetics and Genomics and the Association for Molecular Pathology. Genet Med 2015:17:405-23.

17 Futema M, Bourbon M, Williams M, et al. Clinical utility of the polygenic LDL-C SNP score in familial hypercholesterolemia. Atherosclerosis 2018;277:457-63.

18 Troeung L, Arnold-Reed D, Chan She Ping-Delfos W, et al. A new electronic screening tool for identifying risk of familial hypercholesterolaemia in general practice. Heart 2016;102:855-61.

19 Green P, Neely D, Humphries SE, et al. Improving detection of familial hypercholesterolaemia in primary care using electronic audit and nurse-led clinics. $J$ Eval Clin Pract 2016;22:341-8.

20 Qureshi N, Weng S, Tranter J, et al. Feasibility of improving identification of familial hypercholesterolaemia in general practice: intervention development study. BMJ Open 2016:6:e011734.

21 Kirke AB, Barbour RA, Burrows $\mathrm{S}$, et al. Systematic detection of familial hypercholesterolaemia in primary health care: a community based prospective study of three methods. Heart Lung Circ 2015;24:250-6.

22 Qureshi N, Kai J, Middlemass J, et al. Comparison of coronary heart disease genetic assessment with conventional cardiovascular risk assessment in primary care: reflections on a feasibility study. Prim Health Care Res Dev 2015;16:607-17.

23 Middlemass JB, Yazdani MF, Kai J, et al. Introducing genetic testing for cardiovascular disease in primary care: a qualitative study. Br J Gen Pract 2014;64:e282-9.

24 Khera AV, Emdin CA, Drake I, et al. Genetic risk, adherence to a healthy lifestyle, and coronary disease. N Engl J Med 2016;375:2349-58.

25 Riveros-Mckay F, Weale ME, Moore R, et al. Integrated polygenic tool substantially enhances coronary artery disease prediction. Circ Genom Precis Med 2021:14:e003304.

26 Wald DS, Bestwick JP, Morris JK, et al. Child-parent familial hypercholesterolemia screening in primary care. N Engl J Med 2016;375:1628-37.

27 Lee $\mathrm{C}$, Rivera-Valerio M, Bangash $\mathrm{H}$, et al. New case detection by cascade testing in familial hypercholesterolemia: a systematic review of the literature. Circ Genom Precis Med 2019;12:e002723.

28 Brett T, Qureshi N, Gidding S, et al. Screening for familial hypercholesterolaemia in primary care: time for general practice to play its part. Atherosclerosis 2018;277:399-406.

29 Patel R, Barnard S, Thompson K, et al. Evaluation of the uptake and delivery of the NHS health check programme in England, using primary care data from 9.5 million people: a cross-sectional study. BMJ Open 2020;10:e042963.

30 Battista RN, Blancquaert I, Laberge A-M, et al. Genetics in health care: an overview of current and emerging models. Public Health Genomics 2012;15:34-45. 